\title{
Conceptualization Appropriate Application for Emergency Room Cases for the Unconscious Patients
}

\author{
Evan Asfoura, , Mohammed Alduhaiem, Terkee Alshayae, \\ MBA program/ Collage of business \\ Dar Al Uloom university \\ Riyadh, Saudi Arabia \\ Email:evan@dau.edu.sa
}

Article History: Received: 10 November 2020; Revised 12 January 2021 Accepted: 27 January 2021; Published online: 5 April 2021

\begin{abstract}
:
Applying the new technologies in the health field is growing more and more because of the important effects on the human life. This paper will provide the conceptual model for cloud based application (called Emergency Room (ER) App which support the doctors to improve the diagnostic procedures which will solve an important medical issue at the emergency rooms in case of " an unconscious person". The proposed application will allow storing the sensitive medical information for any person in cloud with high consideration of the privacy aspects. The doctors in the emergency will be able to know all the needed information about the health aspects of the patients directly from the cloud which will be helpful to decide which kind of medicine can be used for recovering the health without any dangerous side effects on those patients.

The result will present two concepts the first one will provide the overview about the health records that should be stored in the cloud and the second provide the workflow which explain how the interaction go among of the actors during the application use.
\end{abstract}

Keywords: Emergency room, ER App, Health, cloud computing,

\section{Introduction}

Using cloud computing applications in health care field is growing clearly during the recent years aiming to provide better care for the beneficiaries of health services (Calabrese and Cannataro, 2015, Nkosi and Mekuria 2010), Ali et al (2018)). Some applications still urgently needed and there is also the need to maintain high numbers in high health indicators is important for the care provided by the Kingdom that is considered the best in the Middle East region. And cloud computing is a technology that relies on transferring the processing and storage space of the computer to the cloud, and it is a server device that is accessed via the Internet, thus transforming information technology programs from products to services, and thus this technology contributes to the sharing of data with the existence and continuous development of information technology programs, Consequently, the efforts of the beneficiaries are focused on using these services only, and the infrastructure for cloud computing depends on advanced data centers that provide large storage spaces for users. It is a web based storage model, where data is stored on multiple virtual servers Instead of hosting it on a single server, and it is usually provided by a third party such as hosting companies that own advanced data centers, they rent cloud storage spaces to their customers according to their needs. The global thought in the field of computer technology and the Internet has changed, and the thought has tended to take advantage of the Internet and provide many of the costs and equipment that those who deal with this technology need, including hardware, maintenance and updating, software, anti-virus and security, and there has become a new thought, cloud computing, to solve many of these problems. 


\section{State of the Art}

Through the literature review. it is clearly no similar concepts to the proposed app in spite of exist apps in $t$ emergency assistance and health. The mentioned apps in the similar field work without internet. Moreover, the proposed app works without any conjunction with other devices like what mentioned in the article by MNK Boulos, AC in the Brewer, and CK that the mobility of a smartphone or tablet allows students to carry a plethora of clinical resources in a convenient and searchable package. According to their article, Apps Also help in monitoring diabeites calculations for patients and reminds them to take their insulin doses. Also, Cree, M.1; Hudson, A.2,,3,,4; Setiyawan, S in 2021 discovered in their research that Twenty-four (9\%) of the 279 MET responses had medication related precipitants primarily megasporogeneses anaphylaxis and opioid related respiratory compromise. One hundred and forty-two (51\%) responses required medications intake. Muhammad Mahtab Alam in 2014 in his survey a new application paradigm life and safety for critical operations and missions using wearable Wireless Body Area Networks (WBANs) technology is introduced. This paradigm has a vast scope of applications, including disaster management, worker safety in harsh environments such as roadside and building workers, mobile health monitoring, ambient assisted living and many more

[Periklis Chatzimisios \& Kan Zheng, 2016] explained that Taking the large number of resource-constrained IoT devices into account, we propose the different kinds of APIs with different application protocols. APIs offer integrations between server-side applications and client-side applications or devices; thus, the specific implementation of APIs on these three parts..

(Paul Patras 2018) fitness trackers record sensitive personal information, including daily step counts, heart rate profiles, and locations visited. By design, these devices gather and upload activity data to a cloud service, which provides aggregate statistics to mobile app users.

In another related work [(Abdullah 2011) aims To handle recent huge production of digital images and data in medical informatics application, the cloud service provider provides services that could be used by medical practitioner for selecting discovering correct information for proper process. Also (Lidong Wang Jul 26th, 2013) was focused on the advantages of cloud computing related to reducing cost and increasing the flexibility which make it useful for providing many healthcare related services to the patients. In this paper, the categories and service models of cloud computing, its technology intelligence, diverse applications in medical services and healthcare, and biometrics-based authentication for information security

JM Kahn, MA Ahuja, and CA Leaver discussed a similar app which is used to make doctors visit online "telemedicine": Telemedicine also has the potential to substantially reduce health care costs. For providers, using telemedicine may be more efficient than seeing patients in brick-and-mortar offices, since it reduces the time and space needed to run a medical practice. Similar to our app, doctors don't need to perform certain test if the once available are recent.

No studies to date have attempted to identify and validate the particular components of the important of such a technology that could involves an app that could save lives if health data is shared with health staffs at the right critical time. (Jochen Brich, Marius Steiert, Michel Rijntjes, 2018). The proposed app in this work also designed to help in many cases that deals with people with learning disabilities, as it saves valuable time to determine his health issue instead of trying to ask the patient or run medical tests that could take valuable time (Elspeth Bradley and Jodi Lofchy. 2018)

\section{Research Methodology}

This paper aim to provide conceptual model which consist of two concepts, the first one about the cloud content related to the patient health records (see figure 1) and the second explains the workflow interaction between the actors in realtime use of the app.(see figure 2).

In addition, figure 1 shows tools the doctors have such as B/S Clients which allow them to add or modify patients reports and the same doctor's reports only. Which means more security to patients health reports. The figure1 also shows the mechanism which doctors will receive an authorization message to his cell phone 
registered in MoH data to all for his/her access. MoH has tools such as sharing data with other applications under the umbrella of Saudi Ministry of Health.

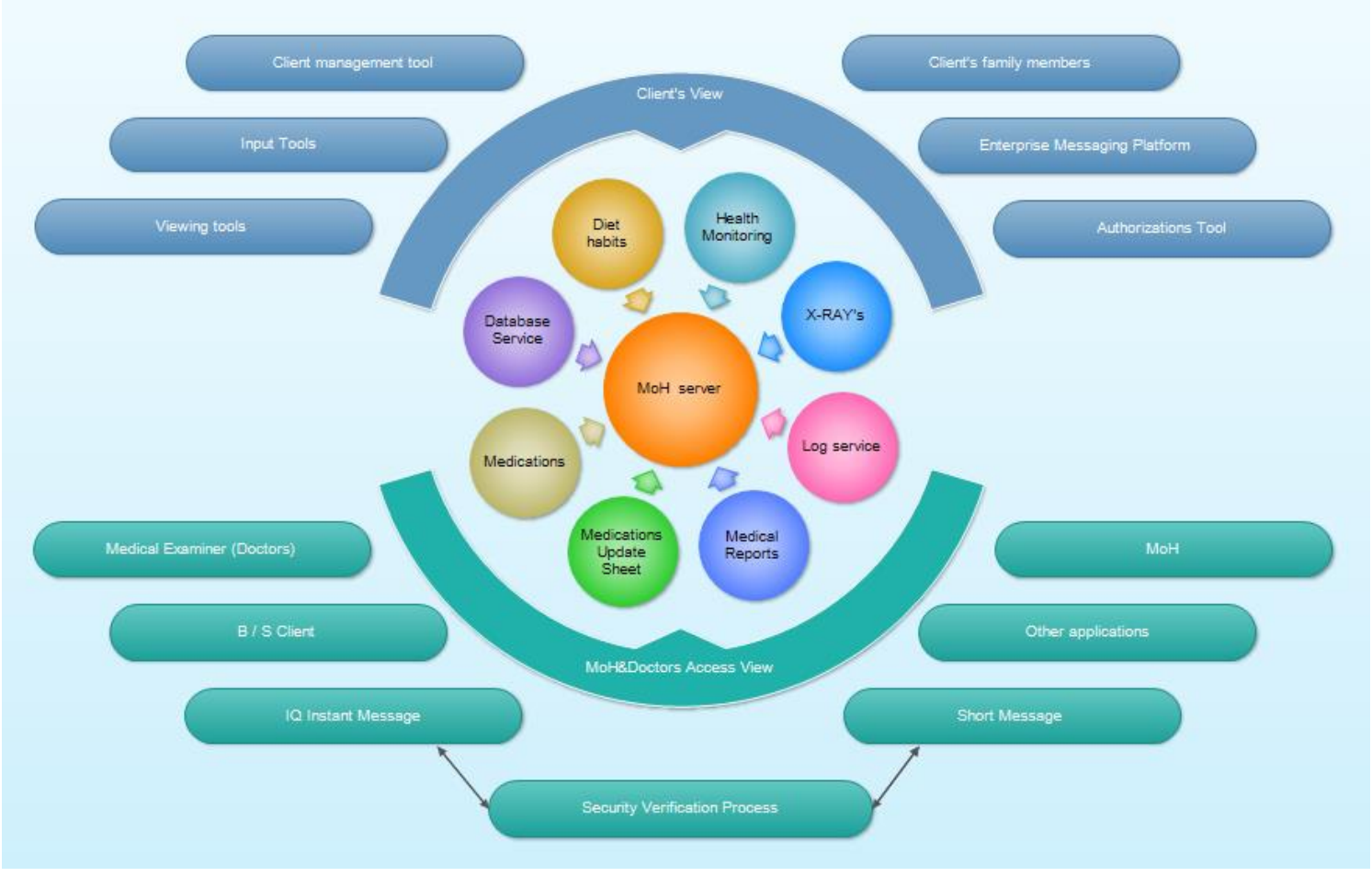

Figure 1: ER App context diagram

Figure 2 shows how the App work and the roles of the actors during the work flow which provided by using sequence diagram including the actors and the interactions between them. The scenario for using the ER App will be as the following:

When the doctor in the emergency receives the patient he will use the ID card of the patient to enter the ID number unconscious with his ID number which has been already registered by $\mathrm{MOH}$.

MOH will check the authority of the Doctor to enter and see the health records of the patient.

The doctor will receive one use number on his mobile to be able to enter the database and see the health records that are important to be considered by the doctor to treat the patient and to know which types of medicine can be used or can be not use in the case of this patient. 


\section{UML Sequence Diagram}

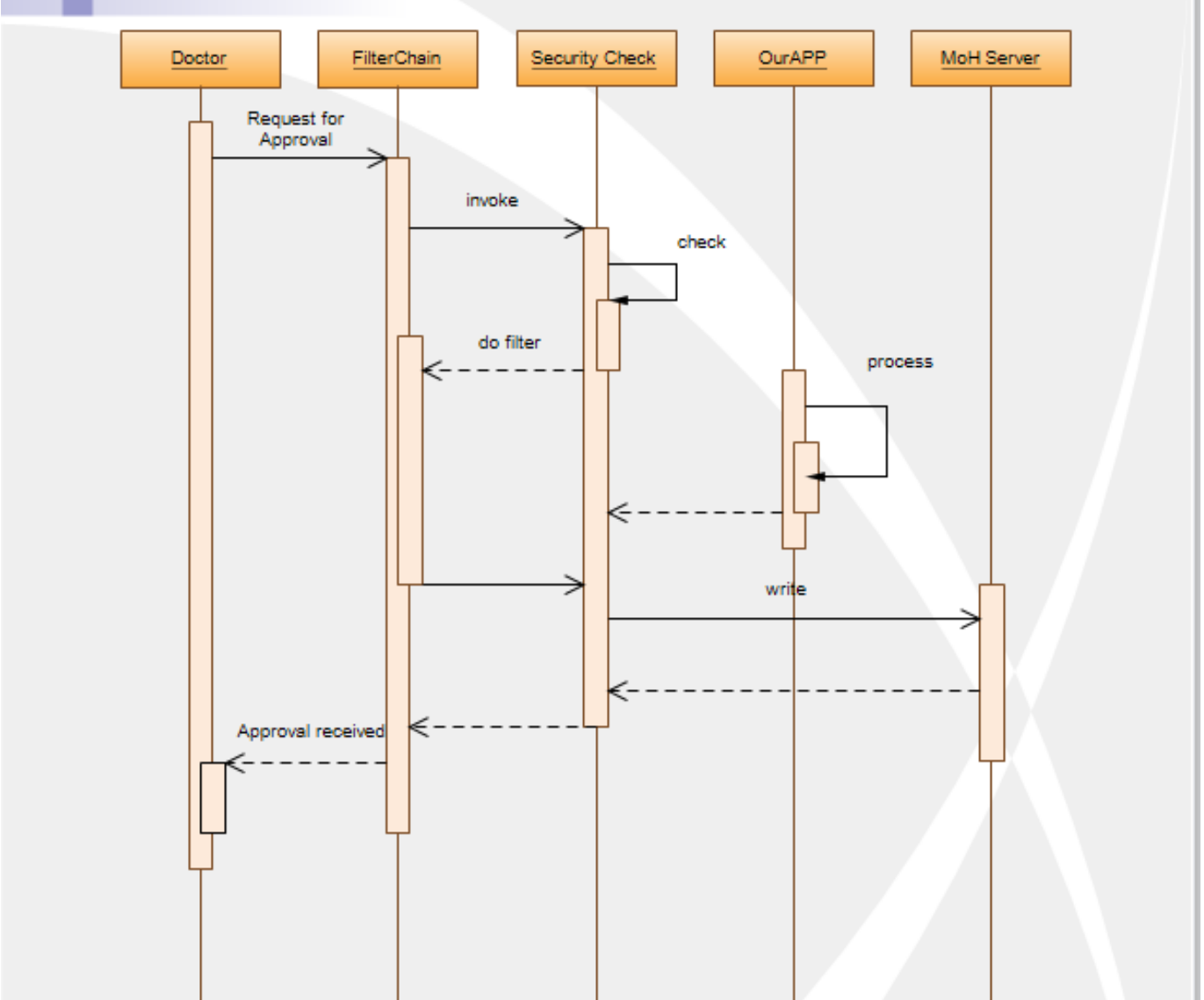

Figure 2: ER App Workflow

ER App advantages

1- The user has free storage space in cloud for his health records.

2- the stored data in cloud spaces will be useful for many application for health care and diagnosing medical situation of the patient in many cases.

3- providing high privacy consideration of the patient medical information where nobody can enter this data except the patient himself and the Doctor who registered in $\mathrm{MOH}$.

4- Upload the files to a file in the cloud itself and run them directly without the need for the Internet

5- When image scanning is done and the system is recognized 6- AI On the chlorize in the meal by searching in browsers

7- Automatic download of any data, even if the programs are different.

8- In order for the files to be shared between the people involved and for ease of use, even when work environments are varied, it is also possible to request to host a link to the files on the mail of the concerned persons.

8- Optional, and anyone can transfer their data to any other platform

9- Connect multiple devices and with any different operating systems

10- The possibility of applying machine learning in the event that sufficient information on patients is available. 


\section{Conclusion}

This work provided the concept of an application which is very important for saving the life of many people who come to the emergency rooms when they are not able to talk to the doctor because they are unconscious. The concept of proposed App includes two diagrams the first one provides overview about the components that the App consist of and the second is sequence diagram for explaining the interactions between the actors (Patient, doctor, system components and $\mathrm{MOH}$ ) in the real time for using the application. The proposed App can be expended to be useful in many health related areas through storage many records related to the health (like sports mode, feeding patterns, work pressures ....etc) which can be analyzed for extracting useful information for supporting the doctor decisions in many cases. The privacy of the patient information has been clearly considered through the identification of the authorities of the persons who are allowed to enter in use the patient data.

\section{Acknowledgement}

The researchers extend their thanks and gratitude to the Deanship of Graduate Studies and Scientific Research at Dar Al Uloom University for their support and funding of this study.

\section{References}

[1]. Cree, M.1; Hudson, A.2,,3,4; Setiyawan, S.( (2021) MEDICATIONS- CAUSE OR SOLUTION FOR MEDICAL EMERGENCY TEAM RESPONSE?, Pediatric Critical Care Medicine: March 2021 - Volume 22 - Issue Supplement 1 3S - p 106 doi: 10.1097/01.pcc.0000738984.12316.36

[2]. Brich, J, Steiert, M, Rijntjes, M. The essential neurological examination of the unconscious patient in the emergency room. Brain Behav. 2018; 8:e01097. https://doi.org/10.1002/brb3.1097

[3]. Boulos, Maged N Kamel et al. "Mobile medical and health apps: state of the art, concerns, regulatory control and certification." Online journal of public health informatics vol. 5,3 229. 5 Feb. 2014, doi:10.5210/ojphi.v5i3.4814

[4]. Kahn, Jeremy. (2015). Virtual Visits - Confronting the Challenges of Telemedicine. The New England journal of medicine. 372. 1684-5. 10.1056/NEJMp1500533

[5]. Bradley, E., \& Lofchy, J. (2005). Learning disability in the accident and emergency department. Advances in Psychiatric Treatment, 11(1), 45-57. doi:10.1192/apt.11.1.45

[6]. Izuddin, Nordin \& Abdullah, Azween \& Issa, Hassan. (2011). Goal-Based Request Cloud Resource Broker in Medical Application. World Academy of Science, Engineering and Technology. 50

[7]. Classen, Jiska \& Wegemer, Daniel \& Patras, Paul \& Spink, Tom \& Hollick, Matthias. (2018). Anatomy of a Vulnerable Fitness Tracking System: Dissecting the Fitbit Cloud, App, and Firmware. Proceedings of the ACM on Interactive, Mobile, Wearable and Ubiquitous Technologies. 2. 1-24. 10.1145/3191737

[8]. Hou, L., Zhao, S., Li, X., Chatzimisios, P., and Zheng, K. (2017) Design and implementation of application programming interface for Internet of things cloud. Int. J. Network Mgmt, 27: e1936. doi: 10.1002/nem.1936.

[9]. Lidong Wang1 , Cheryl Ann Alexander, Medical Applications and Healthcare Based on Cloud Computing, Department of Engineering Technology, Mississippi Valley State University, 2013.

[10]. Jiska Classen, Daniel Wegemer, Paul Patras, Tom Spink, and Matthias Hollick. 2018. Anatomy of a Vulnerable Fitness Tracking System: Dissecting the Fitbit Cloud, App, and Firmware. Proc. ACM Interact. Mob. Wearable Ubiquitous Technol. 2, 1, Article 5 (March 2018), 24 pages. DOI:https://doi.org/10.1145/3191737

[11]. B. Calabrese and M. Cannataro (2015): Cloud Computing in Helthcare and Bioinformatics, in: Scalable Computing: Practice and Experience, Volume 16, Number 1, pp. 1-18. http://www.scpe.org

[12]. M.T. Nkosi and F. Mekuria (2010), Cloud Computing for Enhanced Mobile Health Applications, in proceeding of 2010 IEEE Second International Conference on Cloud Computing Technology and Science, USa, 2010.

[13]. Ali et al (2018): Cloud computing-enabled healthcare opportunities, issues, and applications: A systematic review, in International Journal of Information Management. Volume 43, December 2018, Pages 146-158. 